\title{
Itch: a HECT-type E3 ligase regulating immunity, skin and cancer
}

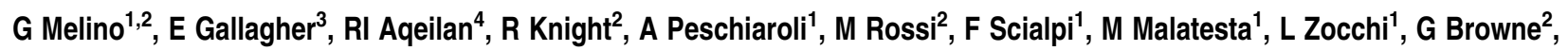 \\ A Ciechanover ${ }^{5}$ and F Bernassola ${ }^{*, 1,6}$
}

The HECT-type E3 ubiquitin ligase (E3) Itch is absent in the non-agouti-lethal $18 \mathrm{H}$ or Itchy mice, which develop a severe immunological disease, including lung and stomach inflammation and hyperplasia of lymphoid and hematopoietic cells. The involvement of Itch in multiple signaling pathways and pathological conditions is presently an area of extensive scientific interest. This review aims to bring together a growing body of work exploring Itch-regulated biological processes, and to highlight recent discoveries on the regulatory mechanisms modulating its catalytic activity and substrate recognition capability. Our contribution is also an endeavor to correlate ltch substrate specificity with the pathological defects manifested by the mutant Itchy mice.

Cell Death and Differentiation (2008) 15, 1103-1112; doi:10.1038/cdd.2008.60

The E3 protein ubiquitin ligase (E3) Itch, or atrophin-1 interacting protein 4 (AIP4, hereafter referred to as Itch), was originally identified through genetic studies aimed to examine the agouti locus, whose mutation results in coat color alterations in mice. ${ }^{1}$ The $18 \mathrm{H}$ mutation, which is associated with a darker coat, arises from a radiation-induced chromosomal inversion that deletes 18 and 20 base pairs from the proximal and distal inversion breaks, respectively. The inversion disrupts the expression of agouti and Itch genes. ${ }^{1}$

The Itch gene encodes 854 amino acids with a relative molecular weight of $113 \mathrm{kDa}$. Itch is a monomeric protein, which belongs to the homologous to E6-AP carboxy terminus (HECT)-type family of E3s, whose modular structural organization consists of an $\mathrm{N}$-terminal $\mathrm{Ca}^{2+}$-dependent phospholipid-binding $\mathrm{C} 2$ domain, multiple protein-protein interaction WW domains, and a C-terminal HECT domain. ${ }^{2}$ The latter coordinates with cognate E2 ubiquitin-conjugating enzyme (E2), and contains an evolutionary conserved cysteine, which forms thioester complexes with ubiquitin, before final attachment of ubiquitin to the target proteins. ${ }^{3}$ Itch possesses four WW domains and a unique proline-rich motif (PRR) located between residues 195 and 246, display- ing important regulatory functions. Similarly to other HECTtype E3s, Itch WW domains most commonly recognize the Pro-rich PPXY (PY) consensus sequence, though they also interact with phospho-Ser/phospho-Thr followed by a Pro residue. In addition, atypical interactions with unrelated modular domains in the substrate ${ }^{4-6}$ or adaptor proteins ${ }^{7,8}$ have been described.

Although a small fraction of Itch displays a perinuclear distribution overlapping with the trans-Golgi network, the protein is predominantly associated with early and late endosomal compartments and lysosomes. ${ }^{5,9}$ Localization of Itch to the endocytic vesicles is mediated by the $\mathrm{C} 2$ domain.

The non-agouti-lethal $18 \mathrm{H}$ or Itchy mice display severe immune and inflammatory defects and manifest a persistent scratching of the skin. ${ }^{1}$ On the C57BL/6J background, Itch deficiency results in spontaneous development of a late onset and progressively lethal systemic autoimmune-like disease, characterized by lymphoproliferation in the spleen, lymph nodes and medulla of the thymus, and by chronic pulmonary interstitial inflammation. Death, occurring at 6-8 months of age, is likely caused by hypoxia, associated with pulmonary inflammation and alveolar proteinosis.

\footnotetext{
${ }^{1}$ IDI-IRCCS Biochemistry Laboratory, Department of Experimental Medicine and Biochemical Sciences, University of Rome 'Tor Vergata', Via Montpellier 1, Rome 00133, Italy; ${ }^{2}$ Medical Research Council, Toxicology Unit, Hodgkin Building, Leicester University, Lancaster Road, P.O. Box 138, Leicester LE1 9HN, UK; ${ }^{3}$ Royal Free \& University College Medical School, 46 Cleveland Street, London W1T 4JF, UK; ${ }^{4}$ Human Cancer Genetics, Ohio State University, Biomedical Research Tower, Room 1088, 460 West 12th Avenue, Columbus, OH 43210, USA and ${ }^{5}$ Cancer and Vascular Biology Center, The Rappaport Faculty of Medicine and Research Institute, Technion-Israel Institute of Technology, Efron Street, Bat Galim, Haifa 31096, Israel

*Corresponding author: F Bernassola, Department of Experimental Medicine and Biochemical Sciences, University of Rome 'Tor Vergata', Via Montpellier 1, Rome 00133, Italy. Tel: + 3906 72596469; Fax: + 3906 20427290;

E-mail: bernasso@uniroma2.it

${ }^{6}$ Current address: IEO c/o IFOM-IEO Campus, Via Adamello 16, 20139 Milan, Italy.

Keywords: ubiquitin E3 ligase; apoptosis; JunB; p53 family members; Notch

Abbreviations: AICD, activation-induced cell death; c-FLIP , cellular FLICE-inhibitory protein; EGFR, epidermal growth factor receptor; HECT, homologous to E6AP $\mathrm{COOH}$-terminus; JNK, jun N-terminal kinase; ICD, intracellular domain; IL, interleukin; Mekk1, mitogen and extracellular kinase kinase 1; PLC- $\gamma 1$, phospholipase C- $\gamma 1$; PKC- $\theta$, protein kinase $\theta$; PY motif, proline-rich sequence; RING, really interesting new gene; Smurf, SMAD ubiquitylation regulatory factor; Su(dx), suppressor of deltex, TCR, T-cell receptor; TGF $\beta$ R, transforming growth factor- $\beta$ receptor; TNF $\alpha$, tumor necrosis factor- $\alpha$; TH, T-helper lymphocyte

Received 25.2.08; revised 26.3.08; accepted 26.3.08; Edited by P Nicotera
} 
Antigen-stimulated naïve CD4 ${ }^{+}$T-helper $\left(T_{H}\right)$ cells can differentiate into two distinct subsets of effector cells, $T_{H} 1$ and $T_{H} 2$, defined by specific cytokine profiles and distinct immune regulatory functions. $\mathrm{T}_{\mathrm{H}} 2$ cells typically produce interleukin- 4 (IL-4) and counteract extracellular pathogens. Dysregulation of the $T_{\mathrm{H}}$ 2-type response is typically responsible for development and maintenance of asthmatic and allergic diseases such as allergic airway inflammation and atopic dermatitis. In response to anti-CD3 and anti-CD28 costimulation, the Itch null $T$ lymphocytes display increased production of $T_{H} 2$ cytokines (e.g. IL-4 and IL-5), causing biased differentiation of $\mathrm{CD}^{+}{ }^{+}$cells into $\mathrm{T}_{\mathrm{H}} 2$ cells and chronic activation. ${ }^{10}$ As a consequence, the Itchy null mice exhibit a higher level of $\mathrm{T}_{\mathrm{H}^{2}}$ dependent immunoglobulin (Ig) G1 and E subtypes than their normal counterpart. ${ }^{10}$

It is relevant to point out that the phenotypic characterization of the Itchy mice on the JU/Ct or C57BL/10 background has revealed that the bias toward production of $\mathrm{T}_{\mathrm{H}}$ 2-derived cytokines is not the only determining factor in the development of the autoimmune disease. ${ }^{11}$ As recently reported by Parravicini et al., ${ }^{11}$ Itch disruption in $\alpha \beta$ and $\gamma \delta$ T cells causes expansion of B1b lymphocytes leading to IgM elevation, and initiates IgE production, respectively. The expansion of $\mathrm{B} 1 \mathrm{~b}$ cells and elevated Ig levels correlated with itching and other inflammatory symptoms. The availability of different animal models of Itch deficiency will certainly contribute to unveil the complex molecular defects underlying the autoimmune pathology.

With few exceptions, Itch typically regulates the stability of both transmembrane receptors through canonical monoubiquitylation or multiubiquitylation, and intracellular substrates through polyubiquitylation, driving them to lysosomal and proteasomal degradation, respectively. Proteolysis-independent ubiquitylation events have been also ascribed to the E3 activity of Itch. ${ }^{6,12}$ A growing number of new substrates and regulatory pathways along with the functional versatility of Itch have been recently brought to light. By illustrating Itch substrate specificities, here, we review the different biological processes involving its E3 activity. The main features of Itch substrates are summarized in Table 1.

\section{Itch Substrates in Relation to Their Biological Functions}

Regulation of the immune response. Notably, several Itch substrates are central players or modulators of the immune response. By providing a portrait of Itch protein targets, we intend to bring together Itch substrate specificity with its ability to regulate the immune system, and ultimately, with the pathological defects developed by the mutant Itchy mice.

Jun family members: the molecular basis underlying Itchregulated differentiation of $T$ lymphocytes. The identification of $C$-Jun and JunB as two Itch protein substrates $8,10,13$ has shed light on the molecular basis underlying the immunological phenotype of the Itchy mice. JunB and c-Jun contain PY or PXY motifs that serve as binding site for Itch WW domains. Ubiquitin-conjugated C-Jun is mainly localized to lysosomal vesicles and its degradation appears to involve both the lysosomal and the proteasomal pathways. ${ }^{13}$ As a result of Itch-mediated canonical ubiquitylation of its substrate JunB, IL-4 promoter occupancy by this transcription factor is greatly reduced upon T-cell receptor (TCR) stimulation. ${ }^{10}$ As JunB protein selectively accumulates in $\mathrm{T}_{\mathrm{H}} 2$ cells and is involved in $\mathrm{T}_{\mathrm{H}} 2$ cell differentiation through the transcriptional regulation of IL-4 and IL-5 promoters, ${ }^{14,15}$ it represents a good candidate mediating the dysregulation of $\mathrm{CD}_{4}^{+}$ T-lymphocyte function observed in the Itchy mice. Under physiological conditions, Itch-induced degradation of JunB maintains the levels of IL-4 at low concentrations, thus attenuating the $\mathrm{T}_{\mathrm{H}} 2$ differentiation response (Figure 1). On the other hand, aberrant expression of JunB, such as a result

Table 1 Main features of Itch substrates

\begin{tabular}{|c|c|c|c|c|}
\hline Substrate & Function & Biological outcome & $\begin{array}{l}\text { Regulators/ } \\
\text { adaptors }\end{array}$ & References \\
\hline c-Jun & Transcription factor & Regulation of $\mathrm{T}_{\mathrm{H}} 2$ cell differentiation/anergy & JNK, N4BP1 & $8,10,12,49,50$ \\
\hline Jun-B & Transcription factor & Regulation of $\mathrm{T}_{\mathrm{H}} 2$ cell differentiation/anergy & JNK, Fyn, Ndfip1 & $10,12,49,50,54,60$ \\
\hline PLC- $\gamma 1$ & Phospholipase & Regulation of T-cell anergy & Unknown & 16 \\
\hline PKC- $\theta$ & Kinase & Regulation of T-cell anergy & Unknown & 16 \\
\hline Notch & Transcription factor & Regulation of autoimmunity & Numb & 4,20 \\
\hline Gli & Transcription factor & Repression of Hedgehog signaling & Numb & 61 \\
\hline Deltex & Regulator of Notch signals & Regulation of autoimmunity & Unknown & 22 \\
\hline Smad2 & Receptor-activated Smad & Activation of TGF $\beta$ R signaling & Unknown & 11 \\
\hline TIEG1 & Transcription factor & Activation of TGF $\beta$ R signaling & Unknown & 27 \\
\hline P73 & Transcription factor & Regulation of apoptosis, neural development, cancer & N4BP1 & 34,8 \\
\hline P63 & Transcription factor & Regulation of apoptosis, epithelial development, cancer & N4BP1 & 35,8 \\
\hline c-Flip & Apoptosis inhibitory protein & Regulation of apoptosis & JNK & 43 \\
\hline ErbB4 & Growth factor receptor & Epithelial kinase receptor, cancer & Unknown & 48 \\
\hline Endophilin A1 & $\begin{array}{l}\text { Protein of the endocytic } \\
\text { machinery }\end{array}$ & Regulation of EGFR endocytosis & Unknown & 9 \\
\hline CXCR4 & Chemokine receptor & Agonist-dependent sorting of G protein-coupled receptors & Unknown & 5 \\
\hline $\mathrm{Hsr}$ & $\begin{array}{l}\text { Protein of the endocytic } \\
\text { machinery }\end{array}$ & Regulation of cargo sorting & Unknown & 5 \\
\hline $\begin{array}{l}\text { TRPV4, } \\
\text { TRPC4 }\end{array}$ & Cation channels & $\begin{array}{l}\text { Regulation of channel recycling and abundance at the cell } \\
\text { surface }\end{array}$ & Unknown & 6 \\
\hline Itch & E3 ubiquitin ligase & $\begin{array}{l}\text { Control of protein stability, potential regulation of E3 catalytic } \\
\text { activity }\end{array}$ & USP9X/FAM & $8,49,50,55$ \\
\hline
\end{tabular}




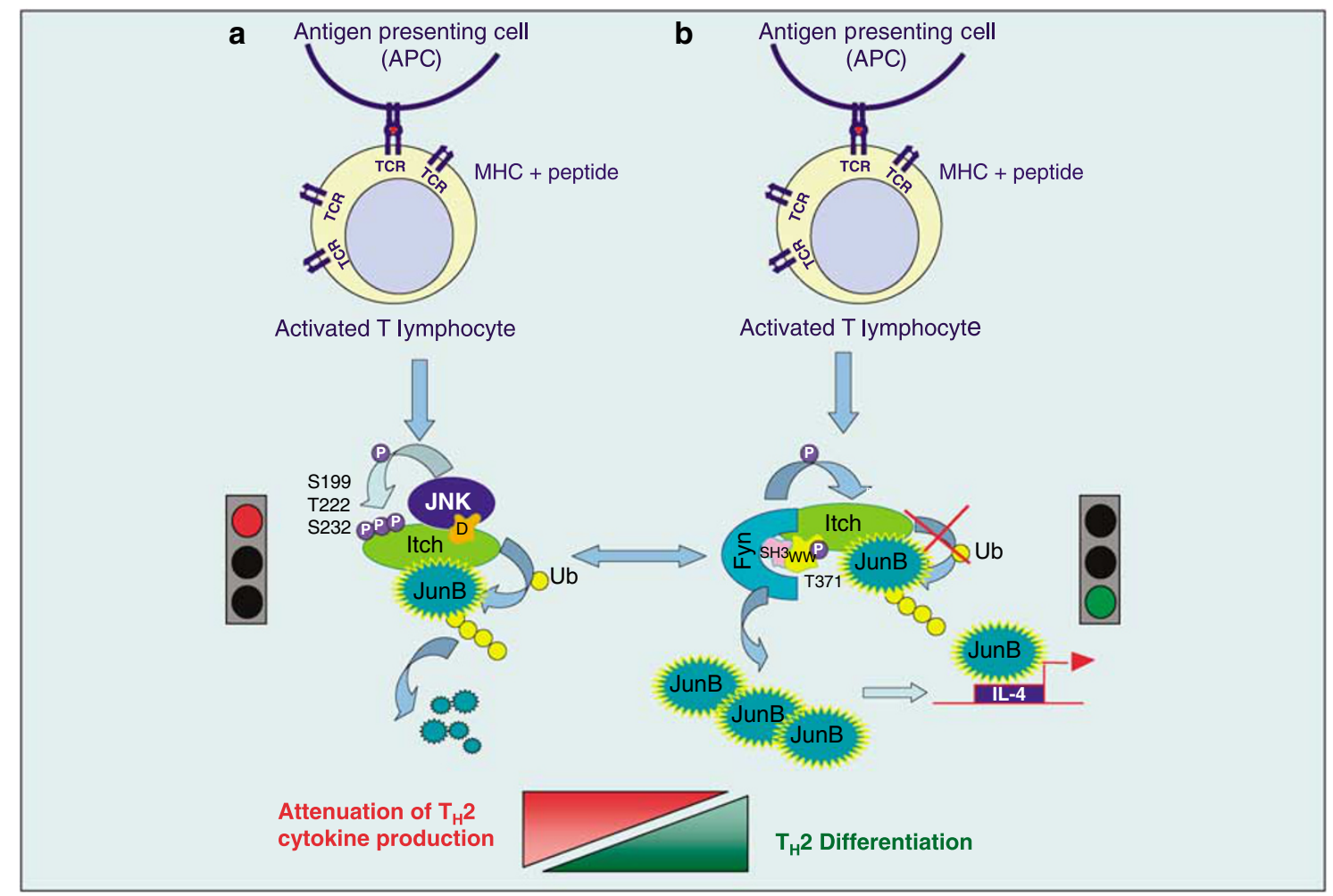

Figure 1 Itch regulation upon TCR engagement. Interaction of a TCR with its cognate antigenic peptide bound to an MHC class II molecule activates a CD4 ${ }^{+}$T-cell response. (a) Attenuation of a $\mathrm{T}_{\mathrm{H}} 2$ response is mediated by the JNK/MAPK signaling. T-lymphocyte stimulation initiates the JNK/MAPK signaling cascade culminating in JunB degradation through the phosphorylation-dependent activation of Itch. Serine/threonine phosphorylation of Itch attenuates the polarization of CD4 ${ }^{+} \mathrm{T}_{\text {-lymphocytes into }} \mathrm{T}_{\mathrm{H}} 2$ effector cells and could prevent JunB from driving IL-4 production in response to strong T-cell activating signals. (b) Potentiation of a $\mathrm{T}_{\mathrm{H}} 2$ response is promoted by the Scr kinase Fyn. An additional level of regulation of Itch activation in response to TCR stimulation is achieved through the Fyn tyrosine kinase signaling pathway. Fyn-catalyzed Tyr371 phosphorylation of Itch prevents its association with JunB. As a consequence of reduced substrate recognition, ubiquitin conjugation and proteasomal proteolysis of JunB are diminished, and augmented IL-4 production accelerates $T_{H} 2$ differentiation. Thus, JunB turnover is finely controlled by upstream kinases through counterbalancing serine/threonine versus tyrosine phosphorylation of Itch

of Itch mutation or downregulation, polarizes $\mathrm{T}$ cells toward the $\mathrm{T}_{\mathrm{H}}$ 2-differentiated features, ultimately causing $\mathrm{T}$-cell hyperproliferation and abnormal allergic responses, including elevated serum $\operatorname{lgG} 1$ and $\operatorname{lgE} .^{10}$ The loss-offunction approach, in which JunB is selectively deleted in T-lymphocytes complements and functionally supports the observation that, enhanced JunB activity due to either Itch loss $^{10}$ or JunB overexpression in mice, ${ }^{14}$ leads to a shift of $\mathrm{CD}^{+}{ }^{+} \mathrm{T}$ cells toward the $\mathrm{T}_{\mathrm{H}} 2$ phenotype. JunB-deficient mice indeed exhibit an impaired allergen-induced airway inflammation. $^{15}$

PLC- $\gamma$ and PKC- $\theta$ : Itch as a T-cell anergy determinant. Induction and maintenance of peripheral immune tolerance is accomplished through the activation of clonal T-cell anergy, a process that prevents the ability of autoreactive lymphocytes to differentiate and proliferate in response to a self-antigen peptide. Anergy induction is triggered by stimulation of the TCR in the absence of the CD28 costimulatory signal on the surface of the antigenpresenting cell (APC), and is typically characterized by the inability of $T$ cells to produce IL-2, even following the restimulation of the TCR and the costimulatory receptor. The establishment of the anergy state requires the $\mathrm{Ca}^{+2}$ / calcineurin-mediated sustained activation of the NFAT transcription factor, in the absence of AP-1 cooperation. Maintenance of T-cell anergy is achieved through the ubiquitin-mediated degradation of key signaling molecules, which ultimately disrupt the integrity of the immunological synapse after restimulation of anergic cells. This aspect has been reviewed at length elsewhere. ${ }^{16}$ The immunological synapse, a supramolecular complex at the T cell/APC interface, mainly composed of adhesion molecules and TCR signal transduction machinery, is crucial for productive T-cell signaling and proliferation.

Itch regulates $\mathrm{T}$-cell anergy maintenance by targeting the phospholipase C- $\gamma 1$ (PLC- $\gamma 1$ ) and the protein kinase $\theta$ (PKC- $\theta$ ), two key signaling molecules induced by calcium/calcineurin signaling, for monoubiquitylation. According to the model proposed by Heissmeyer et al. ${ }^{17}$ Itch mRNA levels are induced during T-cell anergy in an NFAT-dependent manner. After restimulation of anergic cells, newly expressed Itch redistributes from the cytosol to the endosomal compartment, where it can associate with PLC- $\gamma 1$ and PKC- $\theta$, which are adjacent to the immunological synapse. Following Itchmediated monoubiquitylation, PLC- $\gamma 1$ and PKC- $\theta$ undergo endosomal sorting and trafficking into the lysosome for protein degradation. Decrease of PLC- $\gamma 1$ and PKC- $\theta$ abundance is thought to shorten the lifespan of the immunological synapse. 
The inability to sustain stable APC contacts reduces the antigen response of anergic cells and induces T-cell unresponsiveness after TCR engagement. In addition, Itch may regulate anergy and autoimmunity by catalyzing the ubiquitin-dependent degradation of the Jun family members, thus contributing to AP-1 inactivation, and indeed the kinetics of $\mathrm{CD} 3$ versus CD3, and CD28 JunB expression in CD4 ${ }^{+} \mathrm{T}$ cells are different.

The critical role played by Itch in peripheral immune tolerance in vivo became evident from the observation that $\mathrm{T}$ cells from Itch deficient mice are resistant to anergy induction and do not show downregulation of PLC- $\gamma 1$ and PKC- $\theta$ following anergy-stimulating conditions. ${ }^{17}$ Besides the hampering effect exerted on signal transduction at the synapse, Itch may regulate anergy and autoimmunity by catalyzing the ubiquitin-dependent degradation of the Jun family members, thus contributing to AP-1 inactivation. JunB is indeed responsible for AP-1-dependent transactivation of the IL-2 promoter in $\mathrm{T}_{\mathrm{H}} 1$ cells.

Therefore, the inability to induce anergy may be one of the molecular mechanisms underlying the autoimmune symptoms, such as splenomegaly and lymphocyte infiltration, manifested by the Itch deficient mice.

By using an in vivo mouse model of antigen-induced tolerance, Venuprasad et al. ${ }^{18}$ have recently proposed that Itch can also function as a crucial tolerogenic modulator of $\mathrm{T}_{\mathrm{H}} 2$ cells. Under $\mathrm{T}_{\mathrm{H}} 2$ conditions, tolerized Itch null $\mathrm{T}$ cells are more resistant to anergy induction. They indeed do not downregulate the Jun family members, and thereby undergo sustained transcriptional activation of the IL-4 promoter. ${ }^{18}$ As a result, the Itch null mice are thought to develop the autoimmune symptoms, such as the allergic inflammatory lesions affecting the lung.
Notch receptors: highlighting the molecular basis of the Itchy autoimmune disease. The failure to establish peripheral tolerance due to enhanced levels of Jun family members, PLC- $\gamma 1$ and PKC- $\theta$, only in part, accounts for the autoimmune disease observed in the Itch mutant mice. The discovery of Notch as a target of Itch-catalyzed ubiquitylation has provided an additional level of complexity to the regulation of the immune response by Itch $^{4}$. Notch proteins (four in mammals) are evolutionarily conserved type I transmembrane receptors, which mainly function in specifying cell fate decision during differentiation and morphogenesis. Depending on tissue and context, Notch activation can either promote or restrict cell fate determination. During lymphoid development, Notch proteins play an essential role in the induction of $T$ cell and $B$ cell lineages and specification of T-cell effector fates, including $T_{H^{2}}$ differentiation (reviewed in Osborne and Minter ${ }^{19}$ and references therein; Figure 2). In addition, Notch promotes activation and cell survival of mature peripheral $\mathrm{T}$ lymphocytes by upregulating the expression of antiapoptotic proteins such as $\mathrm{Bcl}-2$ and inhibitor of apoptosis proteins (IAPs), and through the activation of AKT (Figure 2). ${ }^{20}$

The ubiquitin/proteasome degradation pathway plays a critical role in regulating Notch signal transduction. Upon ligand binding, Notch proteins undergo a complex proteolytic maturation process, which culminates in the release of the intracellular domain (ICD). Cleavage of the receptor is triggered when the extracellular domain binds to a canonical ligand on a neighboring cell, or upon activation of CD4 ${ }^{+}$ $T$ lymphocytes. The transcriptionally active ICD translocates to the nucleus, where it functions as coactivator to modulate transcription of Notch target genes. Itch-mediated polyubiquitylation of the intracellular portion of membranetethered Notch1 results in the degradation of the ICD following
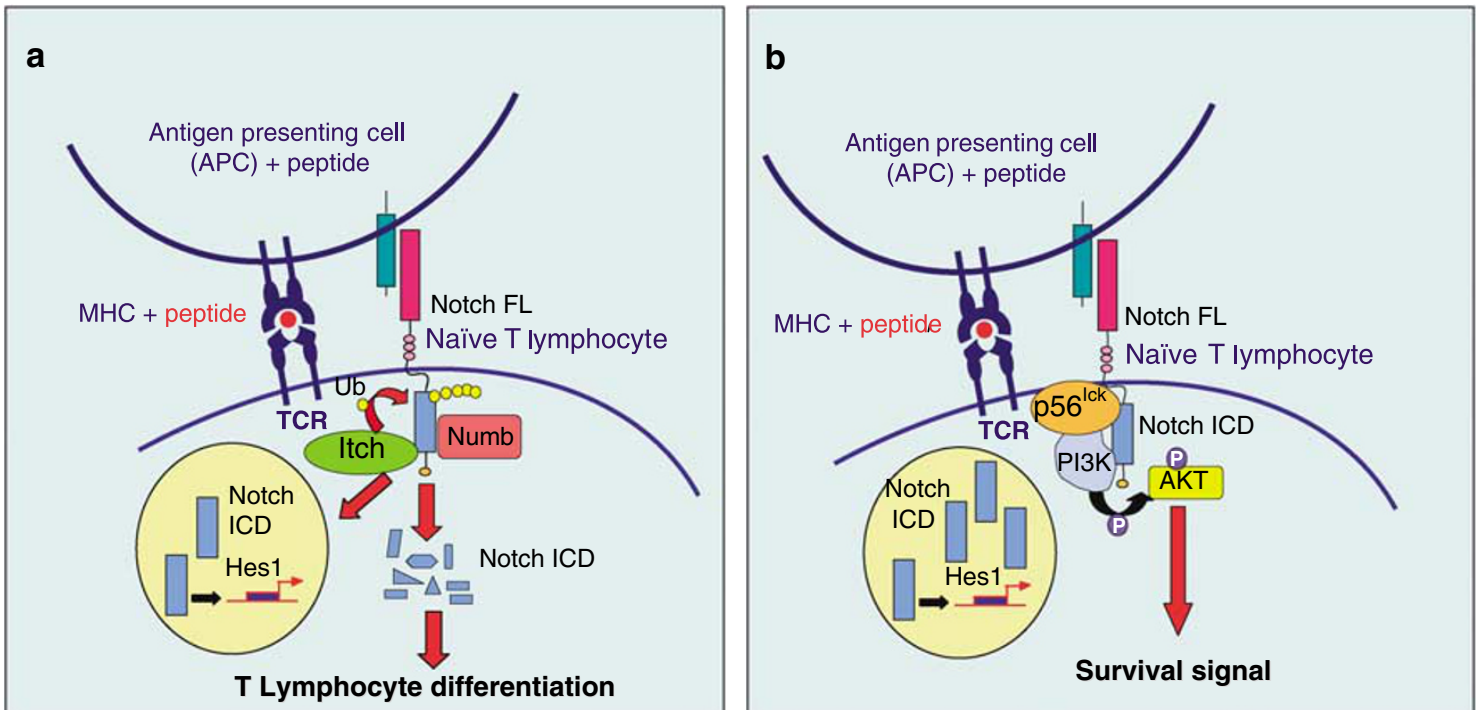

Figure 2 A model for Itch contribution to Notch-regulated lymphoid development. The Notch receptors regulate cell fate decisions during T-cell differentiation and activation. As a result of ligand binding to the extracellular domain, Notch proteins undergo a complex processing mechanism, culminating with the release of the transcriptionally competent ICD from the membrane. (a) Following receptor activation, Itch-mediated ubiquitylation of Notch results in the degradation of the ICD, thus, in turn, antagonizing Notch nuclear activity. The protein adaptor Numb contributes to modulate the endocytic ubiquitin-dependent proteolysis of Notch1 and, thus, to finely tune the specification of T-cell fates determined by Notch signaling. (b) Under physiological conditions, Notch promotes the activation and cell survival of mature peripheral T lymphocytes, by upregulating the expression of a number of antiapoptotic proteins, including AKT. Unbalanced Notch turnover, due to Itch mutation or downregulation, would increase Notch signaling and thereby amplify survival signals leading to abnormal immune responses 
receptor activation (Figure 2). ${ }^{4,21}$ Due to the lack of PY motifs, Notch 1 is atypically recruited by Itch through the $\mathrm{N}$-terminal ankyrin repeat-containing region of the ICD. As discussed later in this review, the binding affinity of Itch for Notch and the resulting receptor degradation are finely tuned by the action of the vertebrate homolog of Drosophila Numb. ${ }^{21}$ An additional level of regulation of Notch activity is accomplished through Itch-mediated degradation of Deltex, which positively controls Notch signaling pathway. Modification of Deltex with lysine29linked polyubiquitin chains labels it for the endocytic pathway, and ultimately, for lysosomal proteolysis. ${ }^{22}$

Notch has been proposed as one of the most relevant Itch target, responsible for the autoimmune phenotype manifested by the Itchy mice. In vivo studies using an activated Notch1 transgene specifically expressed in developing thymocytes showed that increased Notch1 signaling results in a chronic autoimmune-like disease, resembling the Itchy mouse symptoms. ${ }^{23}$ Even more remarkably, the Itchy mutant mice expressing the activated Notch1 transgene, display even more severe pathological lesions including splenomegaly, hepatomegaly and lymphadenopathy, chronic inflammation as well as a significant earlier disease onset. Notch signaling positively regulates the expression of IL- 4 by $\mathrm{CD} 4^{+} \mathrm{T}$ cells. It is, therefore, likely that the hyperproliferative and inflammatory phenotype manifested by the Itchy mice is worsened by further production of IL-4 due to increased expression of Notch in the double-mutant mice. In addition, the mutations in concert yielded a novel phenotype including a perturbation of T-cell development (e.g. negative or positive selection). The severity of the disease correlated with increased Notch1 protein levels, leading to augmented AKT signaling pathway. The survival signal generated through phosphorylated AKT would then allow the persistence of pathological cells that can initiate the autoimmune disease. ${ }^{24}$

Notch signaling and its regulation pathways are evolutionarily conserved mechanisms. The Drosophila mutant Suppressor of deltex $(S u(d x))$ was originally identified as a suppressor of the wing vein thickening caused by $d x$. Phylogenic analysis suggests that $S u(d x)$ is the Drosophila ortholog of mouse Itch. $S u(d x)$ functions as a negative regulator of Notch signaling and interacts with Notch by direct association of its WW domain 3 (WW3) and a PY motif located on the ICD.

Proteolysis-independent roles of Itch in the positive regulation of the TGF- $\beta$ signaling. The intracellular signaling downstream ligand-dependent activation of the transforming growth factor- $\beta$ receptor (TGF $\beta$ R) is tightly controlled by the ubiquitin machinery. ${ }^{25}$ Besides the well acknowledged role of the Smad ubiquitylation regulatory factor (Smurf) E3s in attenuating the TGF- $\beta$ signaling, Itchmediated ubiquitylation of Smad2 positively modulates its phosphorylation by activated TGF $\beta \mathrm{R}^{11}$ Under stimulation conditions, Itch facilitates the interaction between the TGF $\beta$ R and Smad2, and by this means promotes its subsequent phosphorylation in an ubiquitylation-dependent manner.

Among its multiple regulative functions in T-cell homeostasis, TGF $\beta$ R signaling has a critical role in limiting T-cell-mediated autoimmunity, through the maintenance of peripheral Foxp ${ }^{+} \mathrm{CD}^{+}$regulatory $\mathrm{T}_{\text {Reg }}$ cells (reviewed in
Rubtsov and Rudensky ${ }^{26}$ and references therein). This

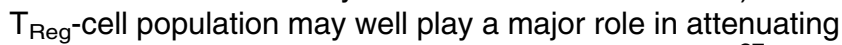
$\mathrm{T}_{\mathrm{H}}$ 2-mediated airway inflammation. Venuprasad et al. ${ }^{27}$ have recently implicated Itch in TGF $\beta$-mediated modulation of Foxp3 expression and generation of $\mathrm{T}_{\text {Reg }}$ cells, through the ubiquitin-dependent transcriptional activation of the TGF $\beta$ inducible early gene 1 product (TIEG1). As Foxp3 is a transcriptional target of TIEG1, Itch ablation severely compromises TGF $\beta$-stimulated Foxp3 expression and causes resistance to TGF $\beta$-induced cell growth inhibition. These discoveries have highlighted a novel mechanism by which tch controls allergic responses by influencing the TGF $\beta$ signaling.

\section{Cell death regulation}

The p53 family members. p73 and p63 share functional similarities with the homologous transcription factor p53, being able to mediate cell cycle arrest and apoptosis in response to DNA damage-induced cellular stress. ${ }^{28-32}$ The tumor suppressor activity of p73 and p63 has been further corroborated by the observation that p73 heterozygous mice spontaneously develop a spectrum of histologically different tumors. $^{33}$

Under normal conditions, p73 and p63 protein levels are generally maintained low through Itch-mediated ubiquitylation. ${ }^{34,35}$ Both transcription factors are recruited by the WW2 of Itch $^{8}$ through a C-terminal PY motif. The PY modules of p73 and p63 span residues 484-487 and 501-504, respectively. ${ }^{34,35}$ Like p53, p73 and p63 accumulate in tumor cell lines in response to $\gamma$-irradiation or treatment with various chemotherapeutic drugs. ${ }^{36}$ In response to genotoxic stress, their induction and transcriptional activation is mainly controlled at the post-translational level, and, at least partially, promoted by DNA damage-caused Itch downregulation. ${ }^{34,37}$ The molecular mechanisms responsible for Itch downregulation following cellular stress, and whether or not aberrant expression or regulation of Itch could negatively regulate the tumor suppressive functions of the p53 family members await further investigation.

E2F1-dependent upregulation of p73 mRNA is a key determinant in the mitochondrial pathway of T-cell apoptosis initiated by the TCR signaling. ${ }^{38-41}$ Hence, another intriguing scenario wherein Itch may play a critical role in regulating p73 protein stability is activation-induced cell death (AICD) of $\mathrm{T}$ lymphocytes. Since, as discussed later in this review, Itch becomes negatively regulated in response to TCR engagement, it is conceivable that stimulation of the receptor would increase p73 protein levels and proapoptotic functions (Figure 3).

While Itch ${ }^{-1-}$ CD4 ${ }^{+}$T cells on the C57BL/6J background exhibit hyperproliferaton following TCR stimulation in vitro and expansion of peripheral populations is observed, it is important to consider that the severity of the $\mathrm{T}_{\mathrm{H}} 2$ bias is background-dependent. ${ }^{30}$ In addition, CD4 ${ }^{+} \mathrm{T}$ cells differentiated under type 1 conditions are significantly more susceptible to AICD than $\mathrm{T}_{\mathrm{H}} 0$ or $\mathrm{T}_{\mathrm{H}} 2$ subsets. ${ }^{42}$ Consequently, our postulation of a role for Itch regulation of p73 driven $\mathrm{CD}^{+}{ }^{+} \mathrm{T}$-cell apoptosis in vivo may be a key feature of the $T_{H} 1$ immune response (Figure 3 ). Indeed, for more complex immune responses, Itch ${ }^{-/-} \mathrm{CD} 4^{+} \mathrm{T}$ cells undergoing $T_{H} 1$ differentiation may well be preferentially deleted 


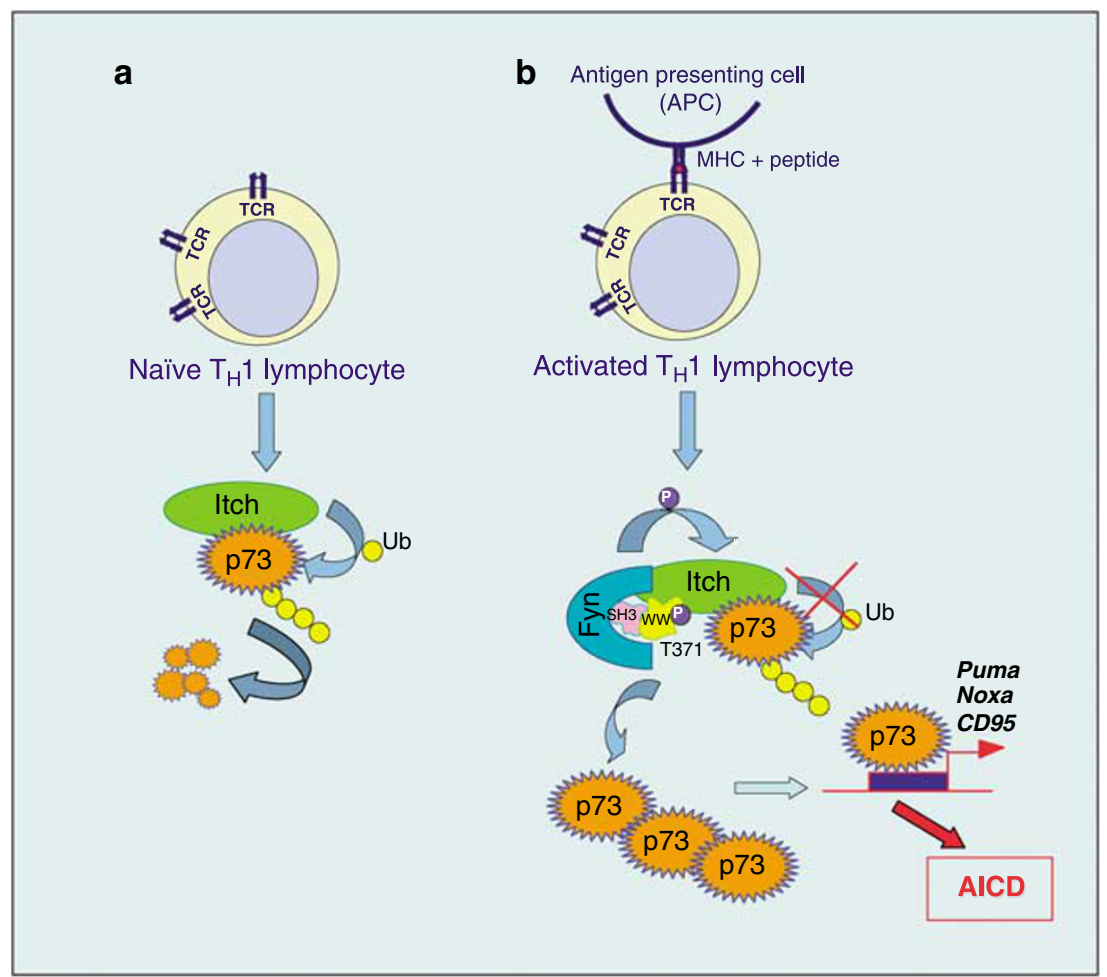

Figure 3 A model for Itch-mediated regulation of activation-induced cell death (AICD). (a) In naïve $T_{H} 1$ lymphocytes, the steady state levels of p73 are kept low by the ubiquitylating activity of Itch. (b) After encounter with specific antigens, post-translational p73 upregulation in $\mathrm{T}_{H} 1$ differentiated subsets of lymphocytes could be the result of (i) Itch downregulation, (ii) transient Tyr-phosphorylation of Itch by Fyn, and (iii) the inhibitory competitive action exerted by N4BP1 on Itch substrate recruitment ability. p73 accumulation in $\mathrm{T}_{\mathrm{H}} 1$ cells leads to increased transactivation of pro-apoptotic target genes

relative to $\mathrm{CD}^{+}{ }^{+}$Itch $^{-1-}$ cells undertaking expansion under $\mathrm{T}_{\mathrm{H}} 2$ differentiation (Figure 1), and could well be a contributing factor to the observation of a CD4 ${ }^{+} \mathrm{T}$ cell/ $\mathrm{T}_{\mathrm{H}} 2$ expansion observed in Itchy mice on the C57BL/6J background. ${ }^{10}$ TNFR1 signaling: cellular FLICE-inhibitory protein (c-FLIP $)$ and receptor interacting protein 1 (RIP1). The involvement of Itch in cell death regulation becomes also evident from its ability to promote tumor necrosis factor- $\alpha$ (TNF $\alpha$ )-induced apoptosis through the proteasomal elimination of the longsplice isoform of the caspase- 8 inhibitor, C-FLIP ${ }_{L} .{ }^{43}$ The amount of cellular C-FLIP $\mathrm{L}_{\mathrm{L}}$ crucially determines the extent of caspase-8 recruitment to the adaptor protein FADD and its rate of activation upon binding of $\mathrm{TNF} \alpha$ to its type 1 receptor (TNFRI). The specificity for the $c-F L I P_{L}$ variant is dictated by the selective ability of a cryptic Itch-binding domain to interact with the C-terminal caspase-8-like (CASP) domain, lacking in the shorter isoform. The in vivo relevance of these findings is uncovered by the observation that, the Itchy mutant mice exhibit protection from fulminant hepatitis, a pathological response to TNF $\alpha$-receptor activation.

More recently, a role for Itch in negatively regulating inflammatory cytokine signaling by forming a functional complex with TAX1BP1 and A20 has been described. This is mediated by Itch binding to two conserved PY motifs in the zinc fingers of TAX1BP1, an activator of A20. Functionally, this novel signaling complex marks RIP1 for inactivation by proteasomal degradation. Consequently, Itch negatively regulates the amplitude of TNFR1, NF- $\kappa \mathrm{B}$, and JNK signaling initiated by RIP1. 44
Regulation of epidermal keratinocyte differentiation. Intriguingly, a number of Itch substrates (c-Jun, JunB, p63, Notch, Gli1) are transcription factors controlling epidermal stem cell maintenance and keratinocyte specification, as well as orchestrating the spatiotemporal progression of terminal differentiation. ${ }^{4,10,35,45}$ It is therefore likely that Itch-mediated degradation of some or all of these proteins would have a regulative role in skin biology.

A key regulator of the expansion of the basal keratinocyte population, as well as of the epidermal terminal differentiation is p63. According to the current model, the $\Delta$ Np63 isoform is responsible for maintaining the basal layer proliferative potential, whereas TAp63 contributes to the stratification and maturation of the suprabasal layers. ${ }^{46}$ p63 and Itch colocalize in the adult human normal epidermis, being predominantly distributed in the basal and the upper layer, respectively. ${ }^{35}$ The in vivo expression gradient of the substrate and the ligase is recapitulated during the in vitro differentiation of keratinocytes, when the accumulation of Itch is paralleled by the reduction of $\Delta$ Np63. ${ }^{35}$ All together, these observations strongly indicate that Itch physiologically controls p63 steady-state protein levels, and, as such, the capacity of the transcription factor to direct the expansion of the basal compartment. ${ }^{35}$

Similarly, Notch receptors have been implicated in governing the balance between proliferative basal cells and terminally differentiating suprabasal epidermal keratinocytes by committing basal progenitors to a spinous cell fate. ${ }^{47}$ In normal epidermis, Notch1-3 receptors are primarily expressed suprabasally, where they negatively regulate the transcription of 

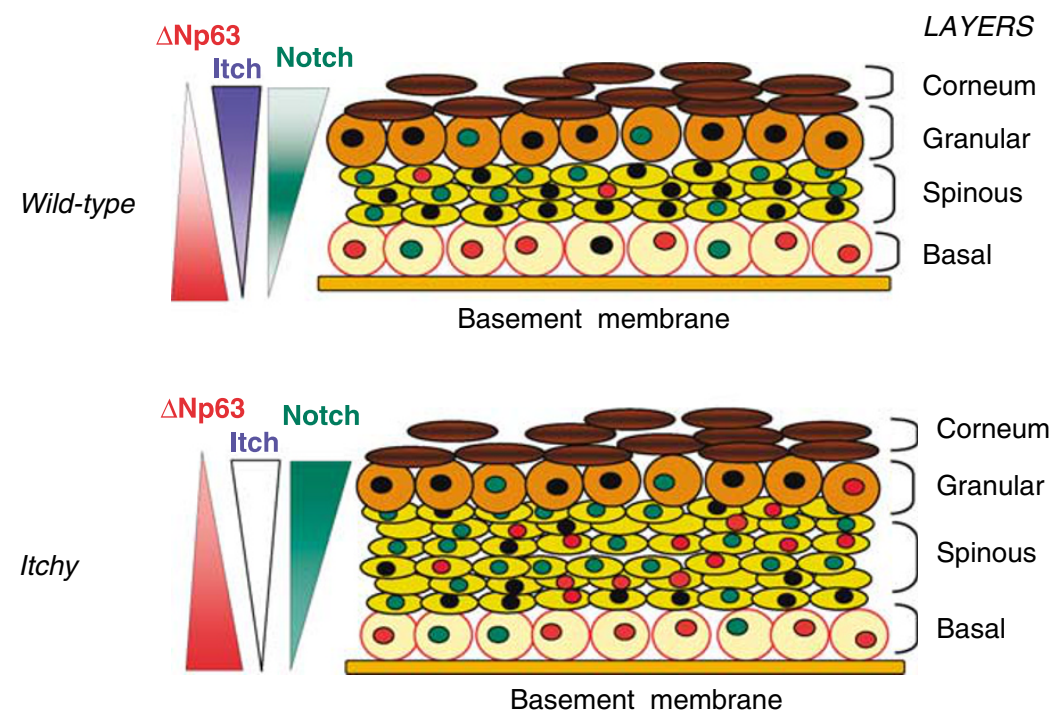

Figure 4 Itch modulates the epidermal keratinocyte differentiation program by targeting multiple substrates for protein ubiquitylation. $\Delta$ Np63 (red triangle), the predominant p63 isoform in the epidermis, is exclusively expressed in the basal proliferative compartment, Notch (green triangle) is mainly expressed in the spinous layer, while Itch (blue triangle) is present throughout the epithelium, though it mainly accumulates in the suprabasal cell layers. By regulating both $\Delta$ Np63 and Notch protein levels, Itch could exert a role in governing epidermal stratification. Itch would facilitate keratinocytes to exit the basal layer by shortening $\Delta$ Np63 half-life in the upper layers and finely tuning Notch expression to promote the basal/spinous transition (upper panel). Lack of Itch would alter the expression of its substrates and lead to epidermal hyperproliferation as a result of an increased number of cells committed to terminally differentiate (lower panel)

genes typically expressed in the basal compartment as well as they activate early genes of the keratinocyte differentiation program. Itch-mediated degradation of Notch would thus contribute to regulate spinous cell fate specification and keratinocyte differentiation (Figure 4).

Abnormal expression of p63 and Notch might be, at least partially, responsible for the increased epidermis thickness phenotype displayed by the Itchy mutant mice (Candi and Melino, unpublished observations, Figure 4). Blanpain et al. ${ }^{47}$ have recently generated a mouse model in which the activated ICD of Notch is constitutively expressed in skin epithelium. Of note, Notch1 ICD transgenic animals display a massive expansion of the spinous layer.

Regulation of receptor trafficking and signaling. A growing body of evidence supports a role for Itch in controlling trafficking of cell signaling receptors at the endosomes. Itch is able to interfere with the endocytic transport at different levels. One such mechanism of action is to direct ligand-activated receptors to the lysosomal degradation pathway.

A recent work by Sundvall et al. ${ }^{48}$ have highlighted a role for Itch in regulating endocytosis and protein stability of ErbB-4, a receptor belonging to the epidermal growth factor receptor (EGFR)/ERbB family. Unique to the ErbB family, following ligand binding, ErbB-4 is proteolytically cleaved to produce a tyrosine kinase IC fragment, which translocates to the nucleus to regulate transcription. In addition to targeting the ICD of ErbB-4 for lysosome-mediated degradation, Itch may interfere with the ErbB-4 signaling by competing with the WW domain-containing transcriptional coactivator Yes-associated protein (YAP) for binding. Cytoplasmic sequestration of ErbB4 by Itch would limit the formation of the transcriptionally active ErbB-4/YAP complexes.
Though a direct effect of Itch on the protein turnover of the EGF tyrosine kinase receptor is still a matter of debate, Itch is thought to promote EGFR trafficking and contribute to EGFR signaling downregulation. Itch utilizes its WW domains to recruit the RING finger E3 Cbl, primarily responsible for the degradation of ligand-stimulated EGFR, and, as a result of this cooperative interaction, Itch would control the EGFR kinase activity. ${ }^{7}$

A further evidence supporting a role for Itch in controlling the EGFR signaling pathway is revealed by the discovery that endophilin-A1, a protein involved in clathrin-mediated endocytosis of the EGFR, is a substrate for endosome-localized Itch. ${ }^{9}$ Lacking the canonical PY recognition motif, endophilinA1 is an additional atypical Itch protein interactor. The unusual nature of this association is achieved through the $\mathrm{SH} 3$ domain of endophilin-A1 and a consensus-binding motif (PXRPPXPR) within the PRR region of Itch. Upon EGFR stimulation, endophilin-A1 and the receptor translocate to the endosomal compartment. In addition, EGFR activation stimulates Itch-mediated endophilin-A1 ubiquitylation, though the precise functional role of this modification in protein trafficking regulation awaits further investigation.

In view of the interference of Itch with the EGFR signaling pathway, it is attractive to speculate that the hyperproliferation of epidermis and stomach epithelium manifested by the Itchy mice $^{1}$ (Candi and Melino, unpublished observations) could also be the result of increased EGFR signaling.

Itch has been also implicated in the agonist-dependent ubiquitylation of the chemokine receptor CXCR4 at the plasma membrane. Ubiquitylated CXCR4 is then targeted for lysosomal degradation. In addition, Itch regulates endosomal sorting of activated CXCR4. Upon receptor internalization, ubiquitylated CXCR4, and Itch traffic to endosomes. In this compartment, Itch mediates the ubiquitylation of Hsr, a protein of the endosomal sorting machinery, which controls ubiquitin-dependent trafficking 
of cargo, including CXCR4, to the degradative pathway. ${ }^{5}$ It has been speculated that conjugation of ubiquitin to Hsr would affect its ability to sort cargo. Hence, Itch-mediated ubiquitylation events would, in turn, promote CXCR4 targeting for lysosomal destruction.

A different mechanism of regulation of the endosomal trafficking by Itch is accomplished by controlling endocytosis and hence the abundance of ion channels at the plasma membrane. Itch-mediated multiubiquitylation of members of the transient receptor potential (TRP) family of cation channels serves as an internalization signal, resulting in downregulation of their cell surface expression and basal activity. ${ }^{6}$ However, once TRP channels undergo ubiquitin conjugation, endocytosed proteins are not degraded, but rather they recycle back to the plasma membrane.

\section{Regulatory Mechanisms Governing Itch Catalytic Activity and Substrate Specificity}

Itch catalytic activity and substrate recognition properties are subjected to many levels of regulation, including several post-translational modifications and interaction with adaptor proteins.

Phosphorylation. Phosphorylation of protein substrates, which facilitates their recognition by the E3s, represents a common regulatory pathway of the ubiquitin conjugation process. Unlike the RING finger-containing E3s, the HECT type ligases typically recognize protein substrates independently of their phosphorylation state. Phosphorylation of the E3 enzymes themselves has been recently emerged as a critical post-translational modification modulating their enzymatic activity or substrate recognition properties. Biochemical and genetic studies have shown that a crucial regulatory mechanism of Itch catalytic activation is Jun N-terminal Kinase1 (JNK1)-mediated Ser/Thr phosphorylation of the E3 upon TCR engagement. ${ }^{49}$ The JNK1 phosphoacceptor sites of Itch have been mapped within the PRR region, where three pro-directed Ser/Thr residues are located. ${ }^{50}$ In response to T-cell activation, JNK1 docking to the $D$ domain, located in the proximal region of the HECT domain (residues 595-604), allows multiple phosphorylation of the E3 on residues Ser199, Thr222, and Ser232. In its unphosphorylated state, Itch enzymatic activity is negatively regulated through intramolecular interactions between the central region, including the WW and PRR motifs, and the C-terminal HECT domain. Following phosphorylation, Itch undergoes a conformational change, which destabilizes the self-inhibitory intramolecular interactions, present within the ligase, thus allowing substrate recruitment and catalytic activation. ${ }^{50}$

Key to the catalytic activity of HECT E3 ligases is the conformational flexibility conferred by a hinge-like region within the bi-lobed HECT structure. ${ }^{51}$ This flexible hinge region is critical for juxtaposing the catalytic cysteine residues of the E2 and the HECT domain, during ubiquitin transfer. Most likely the Itch auto-inhibitory conformation, whereby the PRR and HECT directly interact, prevents such flexibility within the HECT domain, greatly reducing its catalytic activity.

The activation of the JNK1 signaling pathway decreases protein turnover of the Jun family members, thus attenuating the polarization of $\mathrm{CD} 4^{+} \mathrm{T}$ lymphocytes into $\mathrm{T}_{\mathrm{H}} 2$ effector cells (Figure 1). JNK1 can also signal C-FLIP $\mathrm{L}_{\mathrm{L}}$ protein ubiquitylation via a similar phosphorylation-dependent activation of Itch. ${ }^{43}$

These biochemical findings are genetically supported by the phenotypic similarities in $\mathrm{CD}^{+}{ }^{+} \mathrm{T}$ cells between JNK1 null, mitogen and extracellular kinase kinase 1 kinasedeficient (Mekk1) ${ }^{\Delta K D}$ and Itchy mice. ${ }^{10,49,52}$ The Mekk1 ${ }^{\Delta K D}$ mice express an inactive form of MEKK1, an in vivo upstream activator of JNK, ERK and p38 MAPK signaling. ${ }^{53}$ Hampering Itch-mediated degradation of Jun family members, achieved either through Itch mutational inactivation or as a result of JNK1 chemical or peptide inhibition, enhances the expression of $\mathrm{T}_{\mathrm{H}} 2$ cytokine genes (IL-4, 5, 10 and 13) in $\mathrm{CD}^{+}{ }^{+} \mathrm{T}$ cells challenged with $\mathrm{T}_{\mathrm{H}} 2$ polarization conditions. ${ }^{49}$ Hence, the MEKK1-JNK signaling pathway plays a crucial role in the negative regulation of $\mathrm{T}_{\mathrm{H}} 2$ differentiation as well as of peripheral immune tolerance induction. ${ }^{18,49}$

In contrast to the positive regulation induced by the JNK1 pathway, the Src kinase, Fyn, phosphorylates Itch on Tyr 371 residue, leading to the inhibition of its E3 activity. ${ }^{54}$ Transient Tyr phosphorylation of Itch occurs in $\mathrm{T}$ lymphocytes in response to TCR engagement (Figure 1). Fyn binding to Itch is mediated through the interaction of Fyn $\mathrm{SH} 3$ domain with WW3 of Itch. Similarly, Tyr371 is located at the N-terminus of Itch WW3 domain. Following Tyr371 phosphorylation, the association between Itch and its substrates, such as JunB is hampered. Hence, after TCR stimulation, Itch-mediated ubiquitin conjugation to JunB is reduced and the transcriptional factor stabilized. Due to augmented JunB degradation, Fyn-deficient $T$ lymphocytes display a decreased production of $\mathrm{T}_{\mathrm{H}} 2$ cytokines. An additional level of regulation of Fyn activation in response to TCR stimulation could be achieved through the stabilization of $p 73$. In activated $T_{H} 1$ cells, the negative control exerted on Itch may result in increased protein turnover rate and induction of AICD (Figure 3).

The MEKK1-JNK1 and Fyn signaling pathways oppositely regulate the turnover of Jun family members and the immune response by counteracting Ser/Thr and Tyr phosphorylation. These dynamic phosphorylation events, in turn, would result in the attenuation and potentiation of $\mathrm{T}_{\mathrm{H}} 2$ differentiation, respectively (Figure 1 ).

Ubiquitylation against deubiquitylation. Itch undergoes auto-ubiquitylation in vivo. $8,49,50,55$ The molecular nature and the physiological relevance of Itch self-modifications have just begun to be elucidated. It has been recently proposed that, similarly to other E3s, Itch autocatalytic activity negatively controls its protein stability. ${ }^{55}$ Nevertheless, Itch auto-ubiquitylation can also provide a non-proteolytic regulatory function, such as modulating its cellular localization or catalytic activity ${ }^{49,50}$ (Scialpi, Melino, Bernassola, personal communication). Ubiquitin selfconjugation of Itch is subjected to different levels of control, being stimulated by JNK activation ${ }^{50}$ or inhibited by the association with the protein interactor, Nedd4-binding partner-1 (N4BP1), ${ }^{8}$ illustrated later in this review.

Furthermore, Itch is a substrate for the deubiquitylating activity of FAM/USP9X, a member of the ubiquitin-specific proteases (USPs). ${ }^{55}$ By counteracting Itch self-ubiquitylation, FAM/USP9X protects the ligase from proteasomal 
degradation. It could be also possible that FAM/USP9X serves to generate mono-ubiquitylated species, thus adding an additional level of control on the E3.

Interaction with adaptor proteins. The HECT E3s can cooperate with accessory and adaptor proteins, which contribute to modulate their substrate recruiting capacity, subcellular localization, and enzymatic activity (reviewed in Shearwin-Whyatt ${ }^{56}$ and references therein). Itch-interacting proteins enable further specificity to the ubiquitylation reaction. Furthermore, the existence of noncanonical substrates ${ }^{4-6}$ suggests that Itch could be recruited to some of its protein targets through an adaptor molecule.

N4BP1 is a novel Itch negative regulator, acting as a competitor of its substrate recruitment ability. ${ }^{8}$ Although N4BP1 does not contain canonical WW domain docking sites, its binding to these modules determines displacement of the substrates from the ligase. Itch-mediated transfer of ubiquitin to its protein targets is then prevented, and the half-life of the substrates prolonged. As a result, N4BP1 potentiates the transcriptional activity of both p73 and c-Jun. The competition mechanism implies that the selectivity of Itch for the substrates could be modulated either by changes in the affinity of binding to the adaptor molecule, or by alterations of their cellular availability. In this scenario, TCR stimulation-induced accumulation of c-Jun and p73 could be achieved either through N4BP1 induction, or through chemical/conformational modifications, which would enhance the affinity of N4BP1 for the E3.

Nedd4 family interacting protein-1 (Ndfip1) is a membraneassociated protein, originally discovered in an interaction screening using the WW domains of mouse Nedd4. ${ }^{57}$ Ndfip1 possesses two N-terminal PY motifs, which mediate direct interaction with Nedd4 as well as Itch. ${ }^{58}$ In yeast, the Ndfip1 ortholog Bsd2 functions as an adaptor, recruiting the HECT domain E3 Rsp5 to substrates, thus enhancing the recognition and removal of misfolded membrane proteins. ${ }^{59}$ In mammals, the biological outcome of such interaction is promoting itch-mediated degradation of target substrates. ${ }^{60}$ This physical association becomes particularly relevant after T-cell stimulation, when, as a result of Ndfip1 induction, Itch is relocalized from the intracellular vesicles to the inner surface of the plasma membrane. Consequently, enhancement of JunB degradation prevents $\mathrm{T}_{\mathrm{H}} 2$ cytokine production. This functional interaction is corroborated by the phenotype manifested by the Ndfip 1 knockout mice. Similarly to the Itchy mice, Ndfip1 deficient animals are prone to develop a severe skin and lung inflammation, accompanied by hepatomegaly and splenomegaly, and high numbers of infiltrating eosinophils at the inflammatory sites. Lack of Ndfip1 predisposes T lymphocytes toward a $\mathrm{T}_{\mathrm{H}} 2$ phenotype, and biases them toward the production of $\mathrm{T}_{\mathrm{H}} 2$ cytokines.

Another Itch protein interactor exhibiting a regulatory role is the vertebrate homolog of Drosophila Numb. ${ }^{21}$ The mammalian Numb protein, whose phosphotyrosine-binding (PTB) domain recruits the C-terminal ICD of Notch, cooperatively enhances Itch-catalyzed ubiquitylation of the membranebound receptor, and specifically promotes the ICD degradation following receptor activation (Figure 2). This effect is achieved through direct binding of Numb to Itch WW1/2 domains. By promoting rapid degradation of Notch1 ICD,
Numb prevents the translocation of the activated receptor to the nucleus and, ultimately, inhibits Notch-dependent signal transduction.

An additional Itch substrate whose modification by ubiquitin requires the accessory role of Numb is the transcription factor Gli, which mediates the effect of Hedgehog signaling in neural stem cell maintenance and self-renewal. ${ }^{61}$ Numb stabilizes the ligase:substrate complex, and functionally synergizes with the ligase in targeting Gli for ubiquitin-dependent proteasomal proteolysis. Since accumulation of Gli in the nucleus is the major mechanism regulating its transcriptional activation, its lost from the nuclear compartment suppresses the expression of Hedgehog target genes. As such, Numb acts as an antagonistic regulator of Hedgehog signaling during cerebellar development.

The C-terminal WW domains (2-4) of the HECT type E3s are known to directly mediate the association with the substrate providing high affinity binding to the PY motifs..$^{8,62,63}$ A regulatory function has been instead ascribed to the first WW domain. As an example, WW1 of Nedd4 interacts with cofactors that regulate ubiquitin/proteasome-dependent proteolysis of bound substrates. In principle, by interacting with regulatory WW domains of Itch, Numb might act as an adaptor facilitating or stabilizing the interaction between Itch and its substrates.

\section{Conclusion}

The ubiquitin ligase Itch plays key roles in different cellular contexts, in virtue of its functionally distinct substrates. Nevertheless, Itch targets can be simplistically categorized in two main classes: transcription factors and growth factor receptors, some of them acting as gene expression regulators as well. Aberrant accumulation of several signaling proteins such as the Jun family members and Notch due to the loss of Itch protein, critically contributes to the autoimmune phenotype of the Itchy mice. A significant number of the transcriptional regulators targeted by Itch for proteasomal destruction are crucially involved in controlling cell growth, differentiation, and apoptotic processes. This raises the intriguing possibility that their inappropriate removal, due to altered Itch regulation, signaling or activity, would be tightly linked to malignant transformation and chemoresistance.

Acknowledgements. We sincerely apologize for not being able to cite some relevant references because of space constraints. This work has been supported by EU-Grant EPISTEM LSHB-CT-019067; the AIRC Grant 1338; the ISS Grant no. 530/F-A19, Grant Telethon GGP04110, and Philip Morris grant awarded to GM, and by the EU NeOEs Rubicon and NeuroNE, the Israel Science Foundation, the German Israeli Foundation, the Israel Cancer Research Fund, and the Dr. Miriam and Sheldon Adelson Medical Research Foundation awarded to AC.

1. Perry WL, Hustad CM, Swing DA, O'Sullivan TN, Jenkins NA, Copeland NG. The itchy locus encodes a novel ubiquitin protein ligase that is disrupted in $18 \mathrm{H}$ mice. Nat Genet 1998; 18: 143-146.

2. Schwarz SE, Rosa JL, Scheffner M. Characterization of human hect domain family members and their interaction with UbcH5 and UbcH7. J Biol Chem 1998; 273: 12148-12154.

3. Huibregtse JM, Scheffner M, Beaudenon S, Howley PM. A family of proteins structurally and functionally related to the E6-AP ubiquitin-protein ligase. Proc Natl Acad Sci USA 1995; $92: 5249$. 
4. Qiu L, Joazeiro C, Fang N, Wang H-Y, Elly C, Altman $Y$ et al. Recognition and ubiquitination of Notch by Itch, a hect-type E3 ubiquitin ligase. J Biol Chem 2000; 275 35734-35737.

5. Marchese A, Raiborg C, Santini F, Keen JH, Stenmark H, Benovic JL. The E3 ubiquitin ligase AIP4 mediates ubiquitination and sorting of the G protein-coupled receptor CXCR4. Dev Cell 2003; 5: 709-722.

6. Wegierski T, Hill K, Schaefer M, Walz G. The HECT ubiquitin ligase AIP4 regulates the cell surface expression of select TRP channels. EMBO J 2006; 25: 5659-5669.

7. Courbard JR, Fiore F, Adélaïde J, Borg JP, Birnbaum D, Ollendorff V. Interaction between two ubiquitin-protein isopeptide ligases of different classes, CBLC and AIP4/ITCH. J Biol Chem 2002; 277: 45267-45275.

8. Oberst A, Malatesta M, Aqeilan RI, Rossi M, Salomoni P, Murillas R et al. The Nedd4binding partner 1 (N4BP1) protein is an inhibitor of the E3 ligase Itch. Proc Natl Acad Sci USA 2007; 104: 11280-11285.

9. Angers A, Ramjaun AR, McPherson PS. The HECT domain ligase itch ubiquitinates endophilin and localizes to the trans-Golgi network and endosomal system. J Biol Chem 2004; 279: 11471-11479.

10. Fang D, Elly C, Gao B, Fang N, Altman $Y$, Joazeiro $C$ et al. Dysregulation of T lymphocyte function in itchy mice: a role for Itch in TH2 differentiation. Nat Immunol 2002; 3: 281-287.

11. Parravicini V, Field AC, Tomlinson PD, Basson MA, Zamoyska R. Itch-/- \{alpha\}\{beta\} and \{gamma\}\{delta\} T cells independently contribute to autoimmunity in Itchy mice. Blood 2008; e-pub ahead of print.

12. Bai Y, Yang C, Hu K, Elly C, Liu YC. Itch E3 ligase-mediated regulation of TGF-beta signaling by modulating smad2 phosphorylation. Mol Cell 2004; 15: 825-831.

13. Fang D, Kerppola TK. Ubiquitin-mediated fluorescence complementation reveals that Jun ubiquitinated by Itch/AIP4 is localized to lysosomes. Proc Natl Acad Sci USA 2004; 101 4782-14787.

14. Li B, Tournier C, Davis RJ, Flavell RA. Regulation of IL-4 expression by the transcription factor JunB during T helper cell differentiation. EMBO J 1999; 18: 420-432.

15. Hartenstein B, Teurich S, Hess J, Schenkel J, Schorpp-Kistner M, Angel P. Th2 cell-specific cytokine expression and allergen-induced airway inflammation depend on JunB. EMBO J 2002; 21: 6321-6329.

16. Mueller DL. E3 ubiquitin ligases as T cell anergy factors. Nat Immunol 2004; 5: 883-890.

17. Heissmeyer V, Macián F, Im SH, Varma R, Feske S, Venuprasad K et al. Calcineurin imposes $T$ cell unresponsiveness through targeted proteolysis of signaling proteins. Nat Immunol 2004; 5: 255-265.

18. Venuprasad K, Elly C, Gao M, Salek-Ardakani S, Harada Y, Luo JL et al. Convergence of Itch-induced ubiquitination with MEKK1-JNK signaling in Th2 tolerance and airway inflammation. J Clin Invest 2006; 116: 1117-1126.

19. Osborne BA, Minter LM. Notch signaling during peripheral T-cell activation and differentiation. Nat Rev Immunol 2007; 7: 64-75.

20. Jenkinson EJ, Jenkinson WE, Rossi SW, Anderson G. The thymus and T-cell commitment: the right niche for Notch? Nat Rev Immunol 2006; 6: 551-555.

21. McGill MA, McGlade CJ. Mammalian numb proteins promote Notch1 receptor ubiquitination and degradation of the Notch1 intracellular domain. J Biol Chem 2003 278: 23196-23203

22. Chastagner $P$, Israël $A$, Brou $C$. Itch/AIP4 mediates Deltex degradation through the formation of K29-linked polyubiquitin chains. EMBO Rep 2006; 7: 1147-1153.

23. Matesic LE, Haines DC, Copeland NG, Jenkins NA. Itch genetically interacts with Notch1 in a mouse autoimmune disease model. Hum Mol Genet 2006; 15: 3485-3497.

24. Sade H, Krishna S, Sarin A. The anti-apoptotic effect of Notch-1 requires p56lckdependent, Akt/PKB-mediated signaling in T cells. J Biol Chem 2004; 279: 2937-2944.

25. Shi Y, Massague J. Mechanisms of TGF-beta signaling from cell membrane to the nucleus. Cell 2003; 113: 685-700.

26. Rubtsov YP, Rudensky Al. TGF signalling in control of T-cell-mediated self-reactivity. Nat Rev Immun 2007; 7: 443-453.

27. Venuprasad K, Huang H, Harada Y, Elly C, Subramaniam M, Spelsberg T et al. The E3 ubiquitin ligase Itch regulates expression of transcription factor Foxp3 and airway inflammation by enhancing the function of transcription factor TIEG1. Nat Immuno/ 2008; 9 . 245-253.

28. Melino G, De Laurenzi V, Vousden KH. p73: Friend or foe in tumorigenesis. Nat Rev Cancer 2002; 2: 605-615.

29. Melino G, Lu X, Gasco M, Crook T, Knight RA. Functional regulation of p73 and p63: development and cancer. Trends Biochem Sci 2003; 28: 663-670.

30. Irwin MS, Kondo K, Marin MC, Cheng LS, Hahn WC, Kaelin Jr WG. Chemosensitivity linked to p73 function. Cancer Cell 2003; 3: 403-410.

31. Bergamaschi D, Gasco M, Hiller L, Sullivan A, Syed N, Trigiante G et al. p53 polymorphism influences response in cancer chemotherapy via modulation of $p 73$-dependent apoptosis. Cancer Cell 2003; 3: 387-402.

32. Gressner O, Schilling T, Lorenz K, Schulze Schleithoff E, Koch A, Schulze-Bergkamen H et al. TAp63 alpha induces apoptosis by activating signaling via death receptors and mitochondria. EMBO J 2005; 24: 2458-2471.

33. Flores ER, Sengupta S, Miller JB, Newman JJ, Bronson R, Crowley D et al. Tumor predisposition in mice mutant for p63 and p73: evidence for broader tumor suppressor functions for the p53 family. Cancer Cell 2005; 7: 363-373.

34. Rossi M, De Laurenzi V, Munarriz E, Green DR, Liu YC, Vousden KH et al. The ubiquitinprotein ligase Itch regulates p73 stability. EMBO J 2005; 24: 836-848.
35. Rossi M, Aqeilan RI, Neale M, Candi E, Salomoni P, Knight RA et al. The E3 ubiquitin ligase Itch controls the protein stability of p63. Proc Natl Acad Sci USA 2006; 103 12753-12758.

36. Flores ER, Tsai KY, Crowley D, Sengupta S, Yang A, McKeon F et al. p63 and p73 are required for p53-dependent apoptosis in response to DNA damage. Nature 2002; 416: 560-564.

37. Hansen TM, Rossi M, Roperch JP, Ansell K, Simpson K, Taylor D et al. Itch inhibition regulates chemosensitivity in vitro. Biochem Biophys Res Commun 2007; 361: 33-36.

38. Lissy NA, Davis PK, Irwin M, Kaelin WG, Dowdy SF. A common E2F-1 and p73 pathway mediates cell death induced by TCR activation. Nature 2000; 407: 642-645.

39. Irwin M, Marin MC, Phillips AC, Seelan RS, Smith DI, Liu W et al. Role for the p53 homologue p73 in E2F-1-induced apoptosis. Nature 2000; 407: 645-648.

40. De Laurenzi VD, Catani MV, Terrinoni A, Corazzari M, Melino G, Costanzo A et al. Additional complexity in p73: induction by mitogens in lymphoid cells and identification of two new splicing variants epsilon and zeta. Cell Death Differ 1999; 6: 389-390.

41. Wan YY, DeGregori J. The survival of antigen-stimulated T cells requires NFkappaBmediated inhibition of p73 expression. Immunity 2003; 18: 331-342.

42. Varadhachary AS, Perdow SN, Hu C, Ramanarayanan M, Salgame P. Differential ability of T cell subsets to undergo activation-induced cell death. Proc Natl Acad Sci USA 1997; 94 5778-5783.

43. Chang L, Kamata H, Solinas G, Luo JL, Maeda S, Venuprasad K et al. The E3 ubiquitin ligase itch couples JNK activation to TNF alpha-induced cell death by inducing c-FLIP(L) turnover. Cell 2006; 124: 601-613.

44. Shembade N, Harhaj NS, Parvatiyar K, Copeland NG, Jenkins NA, Matesic LE et al. The E3 ligase Itch negatively regulates inflammatory signaling pathways by controlling the function of the ubiquitin-editing enzyme A20. Nat Immunol 2008; 9: 254-262.

45. Candi E, Schmidt R, Melino G. The cornified envelope: a model of cell death in the skin. Nat Rev Mol Cell Biol 2005; 6: 328-340.

46. Candi E, Rufini A, Terrinoni A, Dinsdale D, Ranalli M, Paradisi A et al. Differential roles of p63 isoforms in epidermal development: selective genetic complementation in p63 null mice. Cell Death Differ 2006; 13: 1037-1047.

47. Blanpain C, Lowry WE, Pasolli HA, Fuchs E. Canonical notch signaling functions as a commitment switch in the epidermal lineage. Genes Dev 2006; 20: 3022-3035.

48. Sundvall M, Korhonen A, Paatero I, Gaudio E, Melino G, Croce CM et al. Isoform-specific monoubiquitination, endocytosis, and degradation of alternatively spliced ErbB4 isoforms. Proc Natl Acad Sci USA 2008; 105: 4162-4167.

49. Gao M, Labuda T, Xia Y, Gallagher E, Fang D, Liu YC et al. Jun turnover is controlled through JNK-dependent phosphorylation of the E3 ligase Itch. Science 2004; 306: 271-275.

50. Gallagher E, Gao M, Liu YC, Karin M. Activation of the E3 ubiquitin ligase Itch through a phosphorylation-induced conformational change. Proc Natl Acad Sci USA 2006; 103 1717-1722.

51. Verdecia MA, Joazeiro CA, Wells NJ, Ferrer JL, Bowman ME, Hunter $T$ et al. Conformational flexibility underlies ubiquitin ligation mediated by the WWP1 HECT domain E3 ligase. Mol Cell 2003; 11: 249-259.

52. Dong C, Yang DD, Wysk M, Whitmarsh AJ, Davis RJ, Flavell RA. Defective T cell differentiation in the absence of Jnk1. Science 1998; 282: 2092-2095.

53. Gallagher E, Enzler T, Matsuzawa A, Anzelon-Mills A, Otero D, Holzer R et al. Kinase MEKK1 is required for CD40-dependent activation of the kinases Jnk and p38, germinal center formation, B cell proliferation and antibody production. Nat Immunol 2007; 8: 57-63.

54. Yang C, Zhou W, Jeon M-S, Demydenko D, Harada Y, Zhou H et al. Negative regulation of the E3 ubiquitin ligase itch via Fyn-mediated tyrosine phosphorylation. Mol Cell 2006; 21: 135-141.

55. Mouchantaf R, Azakir BA, McPherson PS, Millard SM, Wood SA, Angers A. The ubiquitin ligase itch is auto-ubiquitylated in vivo and in vitro but is protected from degradation by interacting with the deubiquitylating enzyme FAM/USP9X. J Biol Chem 2006; 281 : 38738-38747.

56. Shearwin-Whyatt L, Dalton HE, Foot N, Kumar S. Regulation of functional diversity within the Nedd4 family by accessory and adaptor proteins. Bioassays 2006; 28: 617-628.

57. Jolliffe CN, Harvey KF, Haines BP, Parasivam G, Kumar S. Identification of multiple proteins expressed in murine embryos as binding partners for the WW domains of the ubiquitin-protein ligase Nedd4. Biochem J 2000; 351: 557-565.

58. Harvey KF, Shearwin-Whyatt LM, Fotia A, Parton RG, Kumar S. N4WBP5, a potentia target for ubiquitination by the Nedd4 family of proteins, is a novel Golgi-associated protein. J Biol Chem 2002; 277: 9307-93017.

59. Hettema EH, Valdez-Taubas J, Pelham HR binds the ubiquitin ligase Rsp5 and mediates the ubiquitination of transmembrane proteins. EMBO J 2004; 23: 1279-1288.

60. Oliver PM, Cao X, Worthen GS, Shi P, Briones N, MacLeod M et al. Ndfip1 protein promotes the function of itch ubiquitin ligase to prevent $\mathrm{T}$ cell activation and T-helper 2 cellmediated inflammation. Immunity 2006; 25: 929-940.

61. Di Marcotullio L, Ferretti E, Greco A, De Smaele E, Po A, Sico MA et al. Numb is suppressor of Hedgehog signalling and targets Gli1 for Itch-dependent ubiquitination. Nat Cell Biol 2006; 8: 1415-1423.

62. Shcherbik N, Kumar S, Haines DS. Substrate proteolysis is inhibited by dominant-negative Nedd4 and Rsp5 mutants harboring alterations in WW domain 1. J Cell Sci 2002; 115 1041-1048.

63. Henry PC, Kanelis V, O'Brien MC, Kim B, Gautschi I, Forman-Kay J et al. Affinity and specificity of interactions between Nedd4 isoforms and the epithelial $\mathrm{Na}+$ channel. $J$ Biol Chem 2003; 278: 20019-20028. 